\title{
Pirfenidone ameliorates the formation of choroidal neovascularization in mice
}

\author{
YUEQI BAO ${ }^{1 *}$, LILI HUANG $^{1 *}$, XIAOBO HUANG ${ }^{1}$, CHUANG GAO $^{1}$, YU CHEN $^{1}$, \\ LIUCHENG WU ${ }^{2}$, SHUNXING ZHU ${ }^{2}$ and YU SONG ${ }^{1}$ \\ ${ }^{1}$ Department of Ophthalmology, Affiliated Hospital 2 of Nantong University; \\ ${ }^{2}$ Laboratory Animal Center of Nantong University, Nantong, Jiangsu 226001, P.R. China
}

Received July 19, 2019; Accepted November 18, 2019

DOI: $10.3892 / \mathrm{mmr} .2020 .11007$

\begin{abstract}
The formation and development of choroidal neovascularization (CNV) is accompanied by inflammation and fibrosis. Existing treatments are expensive and can cause irreversible complications. Pirfenidone (PFD) exerts anti-inflammatory and anti-fibrotic effects; however, its applications in the eye remain unclear. Male C57BL/6J mice (aged 6-8 weeks) were used to explore whether PFD can inhibit the formation of laser-induced CNV. The localization of transforming growth factor $\beta_{2}\left(\mathrm{TGF} \beta_{2}\right)$ was determined through immunofluorescent staining. After laser photocoagulation, the vehicle and PFD groups were intravitreally injected with $1 \mu \mathrm{l}$ PBS and $1 \mu 10.5 \%$ PFD, respectively. At day 7 after intravitreal injection, the expression of $\mathrm{TGF} \beta_{2}$ and vascular endothelial growth factor (VEGF) was assessed. Fundus fluorescein angiography was performed to investigate the extent of fluorescence leakage, and the CNV areas were analyzed using a choroidal flat mount. The results demonstrated that, on day 7 after photocoagulation, the expression of TGF $\beta_{2}$ and VEGF was reduced in the experimental group. In addition, fluorescein angiography showed that the leakage area of CNV was significantly smaller in the PFD injection group than those observed in the control and vehicle groups. Moreover, the areas of CNV in the PFD injection group were smaller compared with those reported in the other two injection groups. Histopathological and TUNEL analyses performed on day 28 revealed that there were no notable abnormalities on the layers of the neural retina of PFD-treated mice. In conclusion, intravitreal injection of PFD inhibited the formation of $\mathrm{CNV}$ in mice, likely via the
\end{abstract}

Correspondence to: Dr Yu Song, Department of Ophthalmology, Affiliated Hospital 2 of Nantong University, 6 Haier Lane North Road, Nantong, Jiangsu 226001, P.R. China

E-mail: songyuleye@126.com

*Contributed equally

Key words: choroidal neovascularization, pirfenidone, transforming growth factor $\beta_{2}$, vascular endothelial growth factor downregulation of VEGF and TGF $\beta_{2}$, which did not cause damage to the mouse retina after 28 days of treatment.

\section{Introduction}

Age-related macular degeneration (AMD) is the leading cause of vision loss among individuals aged $>50$ years in industrialized nations (1). Wet AMD is characterized by choroidal neovascularization (CNV), wherein new blood vessels disrupt Bruch's membrane and grow towards the outer retina from the underlying choroid (2). These immature blood vessels are more prone to leaking and bleeding, thus causing severe impairment of visual acuity (3). CNV is a process involving both inflammation and angiogenesis $(3,4)$. Vascular endothelial growth factor (VEGF) inhibitors (ranibizumab, bevacizumab and aflibercept) are the current standard of care for AMD (5). Although anti-VEGF treatment generally stabilizes and improves visual acuity, not all patients benefit from this type of therapy (5). There is evidence suggesting that the inhibition of transforming growth factor $\beta$ (TGF $\beta$ ) may be a novel therapeutic approach in the treatment of CNV $(6,7)$.

TGF $\beta$ is part of a superfamily of peptide growth factors, regulating a wide range of cellular functions during development and maintaining adult tissue homeostasis (8). High expression of TGF $\beta$ is associated with the expression of angiogenic factors and increased new vessel formation (9). Three separate TGF $\beta$ isoforms (TGF $\beta_{1}, \mathrm{TGF} \beta_{2}$ and TGF $\beta_{3}$ ) have been identified in mammals, and share $70-82 \%$ homology at the amino acid level (10). Among them, TGF $\beta_{2}$ is predominant in normal aqueous and vitreous humor $(11,12)$. VEGF is found in numerous ocular tissues of both healthy and diseased eyes, and it plays a critical role in the development of CNV (13). In AMD, there is evidence that TGF $\beta_{2}$ induces the upregulation of VEGF mRNA expression and protein secretion of VEGF by the retinal pigment epithelium (RPE) and choroid cells (14).

Pirfenidone [5-methyl-1-phenyl-2-(1H)-pyridone; PFD] is a non-peptide, low-molecular weight compound. It was initially evaluated as an anti-inflammatory agent, and subsequently developed as an anti-fibrotic drug (15). Its anti-inflammatory effect is attributed to tumor necrosis factor- $\alpha$ (TNF $\alpha)$ (16). Notably, it reduces the proliferation and differentiation of fibroblasts into myofibroblasts by inhibiting key factors in the TGF $\beta$ pathway (17). The safety and efficacy of PFD have been 
clinically evaluated in numerous disorders. In multinational phase 3 trials involving patients with idiopathic pulmonary fibrosis, the administration of PFD significantly reduced disease progression with an acceptable safety profile $(18,19)$. In a randomized, placebo-controlled trial that included 77 patients with diabetic kidney disease, treatment with PFD led to an improvement in the estimated glomerular filtration rate and was not associated with severe adverse effects (20). PFD is applied to the eye in the following clinical situations: Ocular surface diseases; glaucoma filtration surgery; posterior capsular opacification; and post-traumatic proliferative vitreoretinopathy (21).

Nevertheless, the relationship between PFD and CNV has not been investigated. Based on the peak time of CNV development $(22,23)$, day 7 (after laser photocoagulation) was selected to demonstrate the influence of PFD on the formation of CNV and its effect on VEGF.

\section{Materials and methods}

Animals. A total of 63 male C57BL/6J mice (age: 6-8 weeks; weight: $20-25 \mathrm{~g}$ ) were purchased from Shanghai SLAC Laboratory Animal Co., Ltd. The animals were housed in specific cages with ad libitum access to food and water in a room with a 12/12-h light/dark cycle. The humidity and temperature were maintained at $50 \pm 5 \%$ and $23 \pm 1^{\circ} \mathrm{C}$, respectively. All experimental procedures were performed in accordance with the requirements of the Animal Welfare Committee of Nantong University [permit nos. SCXK(Su)2014-0001 and SYXK(Su)2017-0046]. This study adhered to the Association for Research in Vision and Ophthalmology Statement for the Use of Animals in Ophthalmic and Vision Research (24). The research protocol for the use of animals was approved by the Center for Laboratory Animals of Nantong University.

Intravitreal injection. In this experiment, 54 mice were randomly divided into three groups ( $n=18 /$ group): Control, vehicle and PFD. The control group and the remaining 9 mice (normal group) received no treatment. The injury induced by CNV and the potential toxicity of PFD in the control, vehicle and PFD groups were compared to the normal group (4 mice were used in choroidal flat mount experiment and 5 mice were used in the histopathological examination, respectively). As described in a previous experiment (25), an intravitreal injection of $1 \mu 10.5 \%$ PFD (Beijing Kangdini Pharmaceutical Co., Ltd.), or vehicle (0.01 M PBS solution: Sodium chloride, $137 \mathrm{mM}$; disodium phosphate dodecahydrate, $9 \mathrm{mM}$; and sodium dihydrogen phosphate dehydrate, $2.9 \mathrm{mM}$ ) was administered on day 0 to the PFD and vehicle group, respectively. Mice were decapitated at day 7 and 28 following anesthesia (5\% isoflurane).

Laser-induced $C N V$. The induction of CNV was carried out immediately after drug application. Anesthesia was induced in 54 mice (control, vehicle and PFD group) through inhalation of isoflurane (induction: 5\%, maintenance: 1\%), and the pupils were dilated with topical administration of tropicamide phenylephrine eye drops (Santen Pharmaceutical Co., Ltd.). Mice in the normal group $(n=9)$ were not induced. Following mydriasis, the mice were placed on a platform under the slit lamp and a laser-induced CNV model was established due to rupture of the Brunch's membrane, as previously described (26). Laser photocoagulation (532-nm laser, 200-mW, 100-ms duration, $50-\mu \mathrm{m}$ spot size) was performed bilaterally in each mouse. Laser spots were performed in a standard manner around the optic nerve using a slit lamp delivery system (Vision One; Lumenis), with a handheld cover slip used as contact lens. Photocoagulation lesions were performed in a peripapillary distribution at a distance of 1-2 disc diameters from the optic nerve, avoiding major vessels. The appearance of a bubble following laser treatment, which indicates a rupture of the Bruch's membrane, is an important factor in the induction of CNV. Therefore, only burns in which a bubble was produced were included in subsequent experiments. Spots with hemorrhage or absence of a bubble at the laser site were excluded from the analysis. The eye was subsequently coated with an antibiotic eye ointment. Afterwards, the CNV grade was evaluated, as previously described (25). The control group represented laser-induced CNV without an injection of PFD or vehicle

Immunofluorescence. Eyes were enucleated, fixed in $4 \%$ paraformaldehyde for $24 \mathrm{~h}$ at $4^{\circ} \mathrm{C}$, and sectioned into cryosections $(5 \mu \mathrm{m})$ at $-20^{\circ} \mathrm{C}$ to determine the localization of $\mathrm{TGF} \beta_{2}$ using a specific antibody $(27,28)$. The cryosections were blocked with 5\% BSA (Sigma-Aldrich; Merck KGaA) for $2 \mathrm{~h}$ at room temperature and incubated with mouse monoclonal anti-TGF $\beta_{2}$ antibody (1:50; cat. no. ab36495; Abcam) at $4^{\circ} \mathrm{C}$ overnight. The slides were incubated with the secondary antibody, Alexa Fluor ${ }^{\circledR} 488$ donkey anti-mouse IgG H+L (1:200; cat. no. A-21202; Thermo Fisher Scientific, Inc.) for $2 \mathrm{~h}$, and then DAPI for $5 \mathrm{~min}$, both at room temperature. The sections were imaged using a fluorescence microscope (magnification, x200; Olympus Corporation).

Western blotting. The RPE-choroid-sclera complex was extracted from 5 mice in each group on day 7 after intravitreal injection to detect the protein levels of molecules. The tissues were homogenized and solubilized in RIPA lysis buffer (cat. no. R0278; Sigma-Aldrich; Merck KGaA), containing 1\% protease inhibitors and $1 \%$ phosphatase inhibitors (Thermo Fisher Scientific, Inc.). Protein concentration in the supernatant was quantified using a spectrophotometer (NanoDrop ${ }^{\mathrm{TM}}$ 1000; NanoDrop Technologies; Thermo Fisher Scientific, Inc.). The proteins $(25 \mu \mathrm{g})$, according to their concentration and a molecular weight marker, were loaded on a $10 \%$ SDS gel and subjected to SDS-PAGE. Proteins were subsequently transferred to a polyvinylidene difluoride membrane and blocked with $5 \%$ skim milk for $2 \mathrm{~h}$ at room temperature. The membrane was incubated with mouse monoclonal anti-TGF $\beta_{2}$ antibody (1:1,000; cat. no. ab36495; Abcam), VEGF rabbit polyclonal antibody (1:5,000; cat. no. 19003-1-AP; ProteinTech Group, Inc.) and mouse monoclonal antibody against GAPDH (1:2,000; cat.no. AT0002; CMCTAG; Engibody Biotechnology, Inc.) at $4^{\circ} \mathrm{C}$ overnight. Then, the membranes were incubated with horseradish peroxidase-conjugated secondary antibodies (1:2,000; cat nos. SA00001-1 and SA00001-2; ProteinTech Group, Inc.) at $37^{\circ} \mathrm{C}$ for $2 \mathrm{~h}$, and washed in Tris-buffered saline and Tween-20 (20 mM Tris-chloride, $\mathrm{pH}$ 7.5; $137 \mathrm{mM}$ sodium chloride; and $0.1 \%$ Tween-20). The blots were then incubated 
A
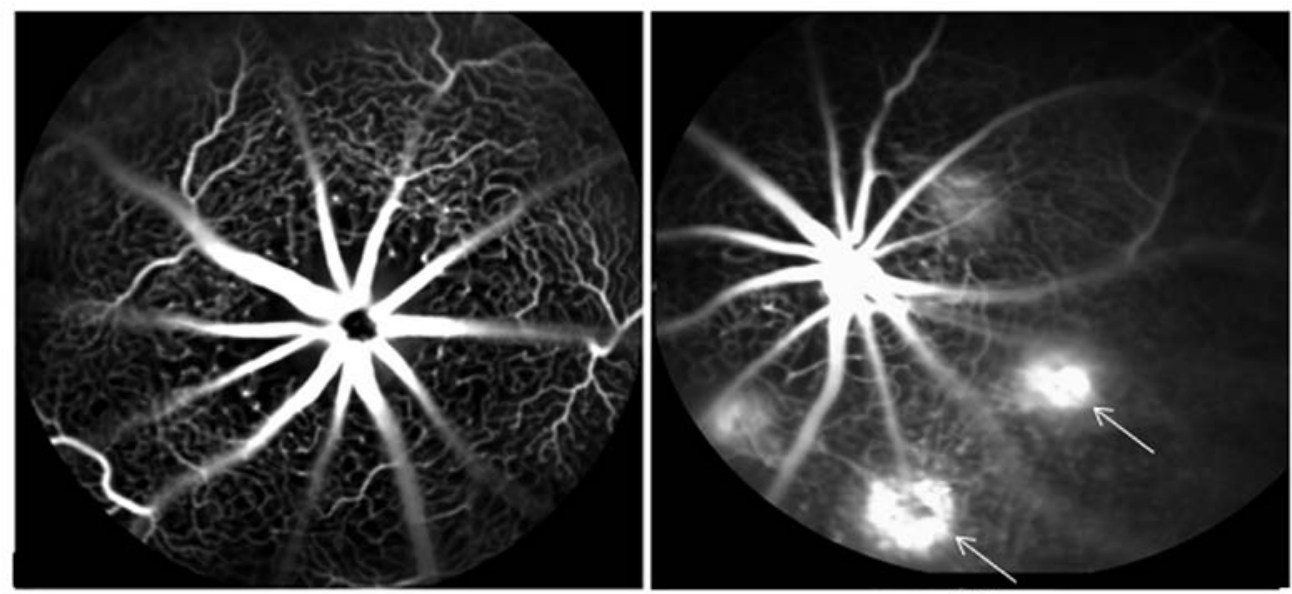

Normal

CNV
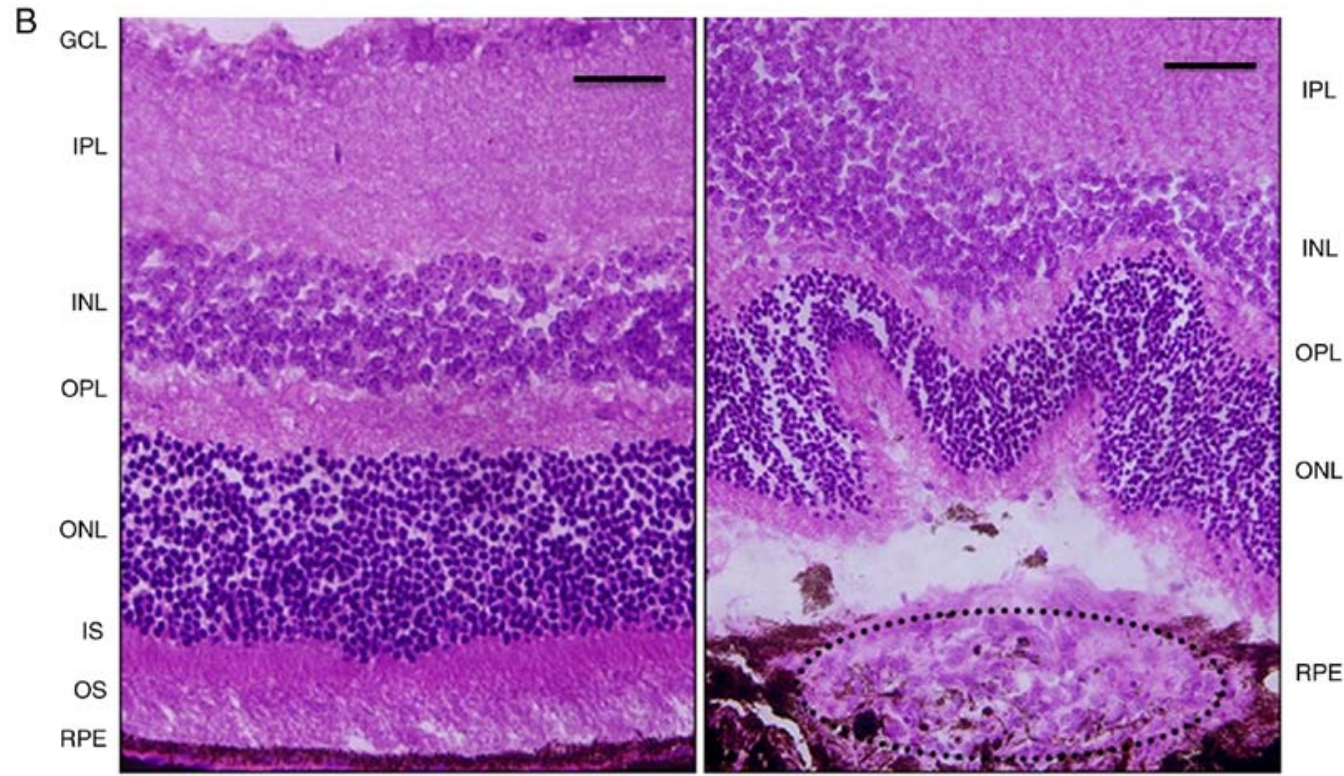

Figure 1. Changes in the mouse retina 1 week after laser photocoagulation. (A) FFA revealed the presence of hyperfluorescence leakage in the laser-induced spots (white arrows) compared with control (magnification, x100). (B) H\&E staining of the mouse retina showed that the fibrovascular complex broke the RPE into the subretinal space after laser photocoagulation compared with control. Scale bar, $50 \mu \mathrm{m}$. RPE, retinal pigment epithelium; OS, outer segment; IS, inner segment; ONL, outer nuclear layer; OPL, outer plexiform layer; INL, inner nuclear layer; IPL, inner plexiform layer; GCL, ganglion cell layer; CNV, choroidal neovascularization; FFA, fundus fluorescein angiography.

with WesternBright ECL (APG Bio, Ltd.) and exposed to an image analysis system (Tanon 5200 Multi; Tanon Science and Technology Co., Ltd.). The intensity of GAPDH was used as the control, and the optical density of bands was quantified using Image J version 1.47 software (National Institutes of Health).

Fundus fluorescein angiography (FFA). At 1 week after laser photocoagulation or intravitreal injection, fundus examinations were performed on 18 mice under general anesthesia. Thereafter, the mice were euthanized as previously described. Mice had their pupils dilated, and a digital fundus camera was used to image the back of the eye (Heidelberg Retina Angiograph II; Heidelberg Engineering, Inc.). The laser-induced lesions were studied using FFA to evaluate the development of CNV. FFA images were captured 2-5 min after intraperitoneal injection of $0.3 \mathrm{ml} 2 \%$ fluorescein sodium (Guangxi Yinzhou People's Pharmaceutical Co., Ltd.) (29). Angiograms were graded as follows: Not stained, score 0; slightly stained, score 1 ; moderately stained, score 2 ; strongly stained, score $3(30,31)$.

Choroidal flat mount. At 1 week after the CNV-inducing laser procedure or intravitreal treatment, 15 mice (10 eyes/group) were euthanized; the eyes were enucleated and fixed in $4 \%$ paraformaldehyde solution for $1 \mathrm{~h}$ at room temperature. The RPE-choroid-sclera complexes were obtained by removing the anterior segments and the neural retina, using an operation microscope (Olympus Corporation). Subsequently, the complexes were washed in PBS containing $0.5 \%$ bovine serum albumin, $0.2 \%$ Tween-20 and 0.1\% Triton-X 100. Eyecups were incubated with fluorescein Griffonia simplicifolia lectin I isolectin B4 (1:100; cat. no. FL-1201; Vector Laboratories Inc.) overnight at $4^{\circ} \mathrm{C}$ to label invading choroid vessels and washed three times in PBS. After staining, the eyecups were flattened through four to six radial cuts from the edge to the equator, and flat mounted with the scleral side facing down onto a microscope slide. The flat mounts were analyzed using a fluorescence 

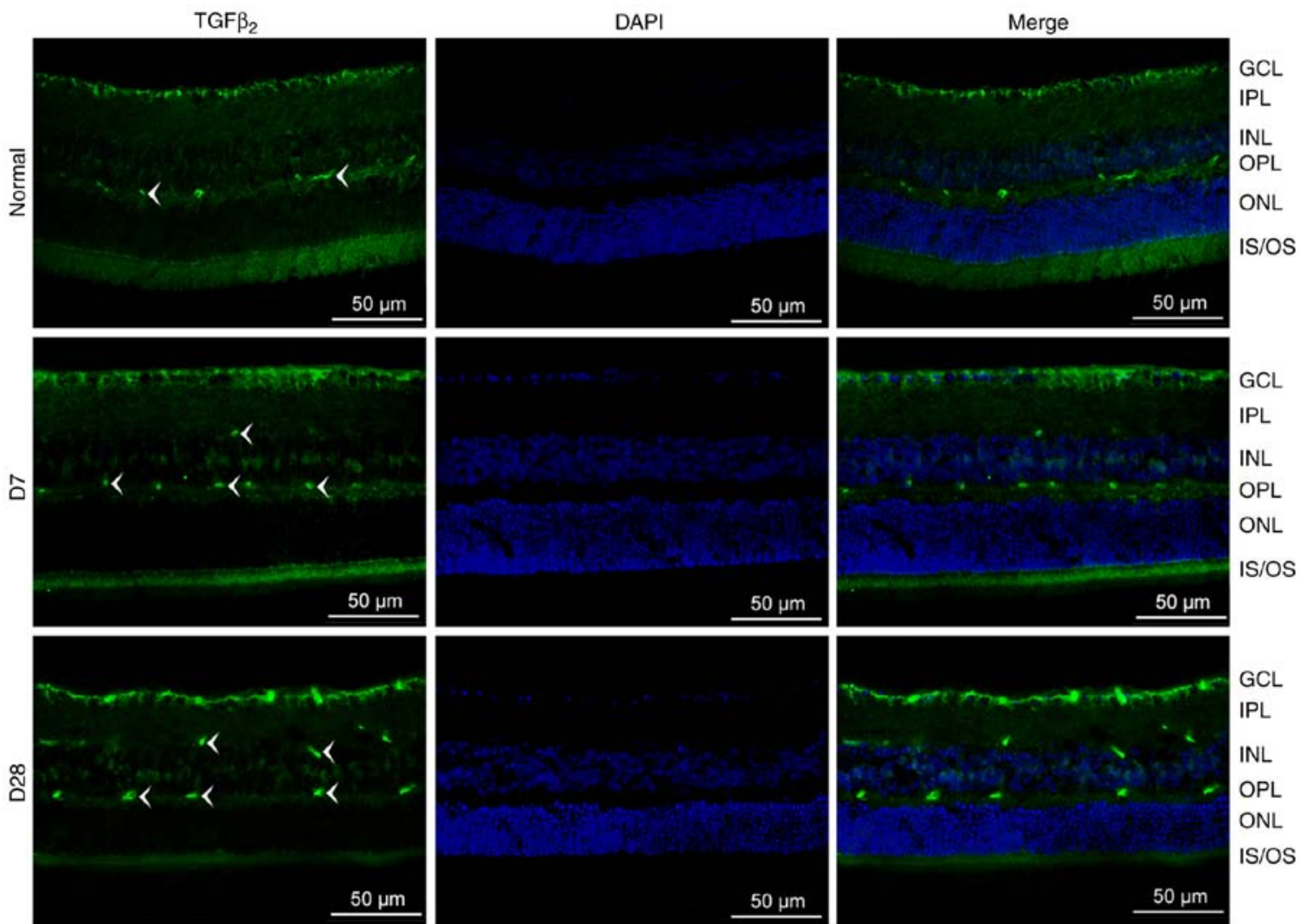

Figure 2. Immunolocalization of TGF $\beta_{2}$ in the mouse retina. Cryosections of the mouse retina were marked with TGF $\beta_{2}$ (green) and cell nuclei were labeled with DAPI (blue). Localization and expression of $\mathrm{TGF}_{2}$ (white arrow heads) in the mouse retina differed between different days. Scale bar, $50 \mu \mathrm{m}$. OS, outer segment; IS, inner segment; ONL, outer nuclear layer; OPL, outer plexiform layer; INL, inner nuclear layer; IPL, inner plexiform layer; GCL, ganglion cell layer; $\mathrm{TGF}_{2}$, transforming growth factor $\beta_{2}$.

microscope (magnification, x100; Olympus Corporation). The size of isolectin B4-positive CNV areas was quantified with Image J software (National Institutes of Health).

Histopathological examination. After the animals were sacrificed, the eyeballs were enucleated, fixed in $4 \%$ paraformaldehyde solution overnight at $4{ }^{\circ} \mathrm{C}$, conventionally dehydrated and embedded in optimal cutting temperature compound to produce cryosections at $-20^{\circ} \mathrm{C}$. The optic nerve parallel to the sagittal plane at the laser photocoagulation position was selected, and slices (thickness, $5.0 \mu \mathrm{m}$ ) were continuously prepared. The sections were stained with hematoxylin-eosin (H\&E) for $3 \mathrm{~min}$ at room temperature, observed, and photographed using a light microscope (magnification, x200; Olympus Corporation).

TUNEL. Cryosections $\left(-20^{\circ} \mathrm{C} ; 5 \mu \mathrm{m}\right)$ were permeabilized with $10 \%$ proteinase $\mathrm{K}$, after fixing in $4 \%$ paraformaldehyde solution at room temperature for $\sim 30 \mathrm{~min}$ according to the manufacturer's protocol (One Step TUNEL Apoptosis Assay Kit; Nanjing KeyGen Biotech Co., Ltd.). Apoptotic cells were treated with terminal deoxynucleotidyl transferase (TdT) enzyme reaction mixture $(1 \mu \mathrm{l}$ TdT enzyme and $4 \mu \mathrm{l}$ biotin-11-2'-deoxyuridine 5'-triphosphate diluted in $1 \mathrm{ml}$ equilibration buffer) at $37^{\circ} \mathrm{C}$ for $45 \mathrm{~min}$ and detected using streptavidin-fluorescein. Subsequently, the slides were washed with PBS three times (15 min each) and sealed with mounting medium (cat. no. 4112APG; Richard-Allan Scientific ${ }^{\mathrm{TM}}$;
Thermo Fisher Scientific, Inc.). Positive and negative controls were included by adding deoxyribonuclease I reaction mixture and omitting the TdT enzyme reaction mixture, respectively. TUNEL-positive cells were observed in $>4$ randomly selected fields under a fluorescent microscope (magnification, x200).

Statistical analysis. All values are presented as the mean \pm SD. One-way ANOVA was used for statistical comparisons between multiple groups. All pairwise multiple comparisons were performed using the Bonferroni test. Descriptive statistics were performed using the SPSS version 22.0 software (IBM Corp.). $\mathrm{P}<0.05$ was used to indicate statistical significance. Each experiment was performed in triplicate.

\section{Results}

Formation of $C N V$ in the mouse retina after laser injury. At 1 week after laser photocoagulation, FFA was performed in normal (without CNV or treatment) and CNV model mice to examine the formation of CNV (Fig. 1A). The laser-induced spots showed hyperfluorescent leakage, indicating rupture of Bruch's membrane and the formation of CNV. Histological analysis of the retina stained with H\&E (Fig. 1B) also confirmed the formation of $\mathrm{CNV}$ on day 7 after laser-induced rupture of Bruch's membrane.

Localization of $\mathrm{TGF}_{2}$ changes in the mouse retina after laser injury. As shown in Fig. 2, $\mathrm{TGF}_{2}$ is expressed in both 
A

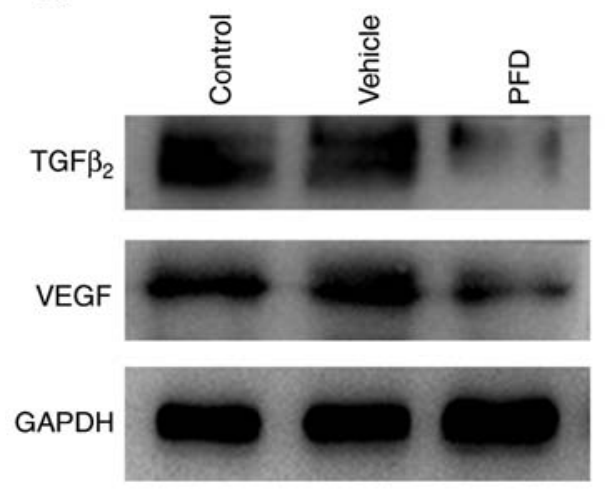

B

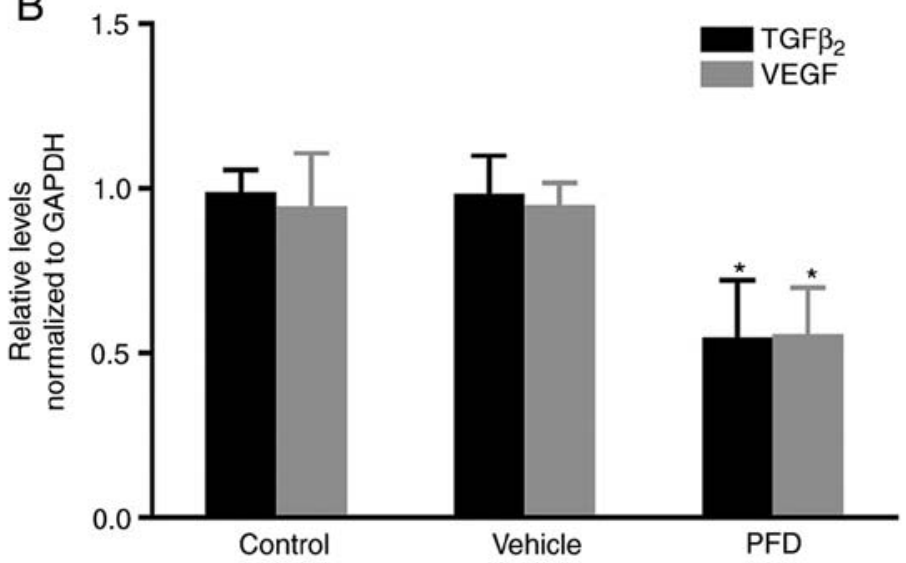

Figure 3. Intravitreal injection of PFD downregulates the expression of TGF $\beta_{2}$ and VEGF. (A) Western blotting showed the expression of TGF $\beta_{2}$ and VEGF in the retinal pigment epithelium-choroid-sclera complex at day 7 after intravitreal injection of PFD or PBS. (B) Quantification graphs for TGF $\beta_{2}$ and VEGF. Data of the relative protein level normalized to that of GAPDH are presented as the mean \pm SD. "P<0.05 vs. vehicle. TGF $\beta_{2}$, transforming growth factor $\beta_{2}$; PFD, pirfenidone; VEGF, vascular endothelial growth factor.

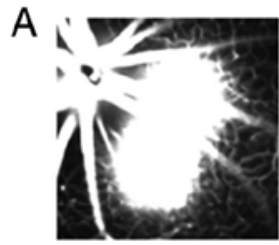

Control

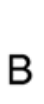

B

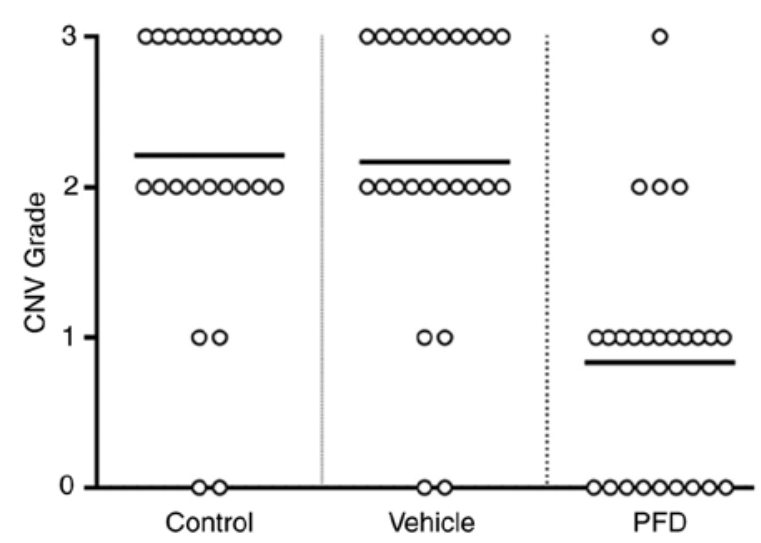

Figure 4. Intravitreal injection of PFD reduces the leakage area of CNV. (A) Fluorescent leakage surrounding the laser spots was relatively weak (score 1) in the PFD injection group compared with the control and vehicle injection groups (magnification, x100). (B) Extent of fundus fluorescein angiography leakage in the control, vehicle and PFD injection groups. ${ }^{* *} \mathrm{P}<0.01$ $(\mathrm{n}=24)$. CNV, choroidal neovascularization; PFD, pirfenidone.

the normal and injured retina, and this expression was significantly increased on day 28 . In the normal mouse retina, TGF $\beta_{2}$ was localized to the ganglion cell layer (GCL) and outer plexiform layer (OPL). After laser photocoagulation (days 7 and 28), TGF $\beta_{2}$ could also be observed in the inner plexiform layer (IPL). The green labels were more intense on day 28 than on day 7 .

PFD suppresses the expression of TGF $\beta_{2}$ and VEGF. The level of TGF $\beta_{2}$ protein in the RPE-choroid complex was
A

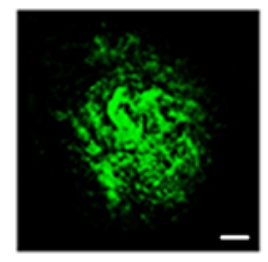

Control

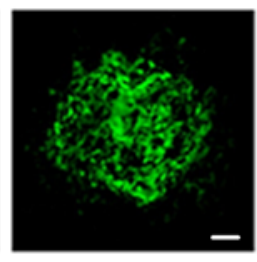

Vehicle

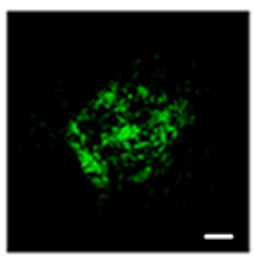

PFD

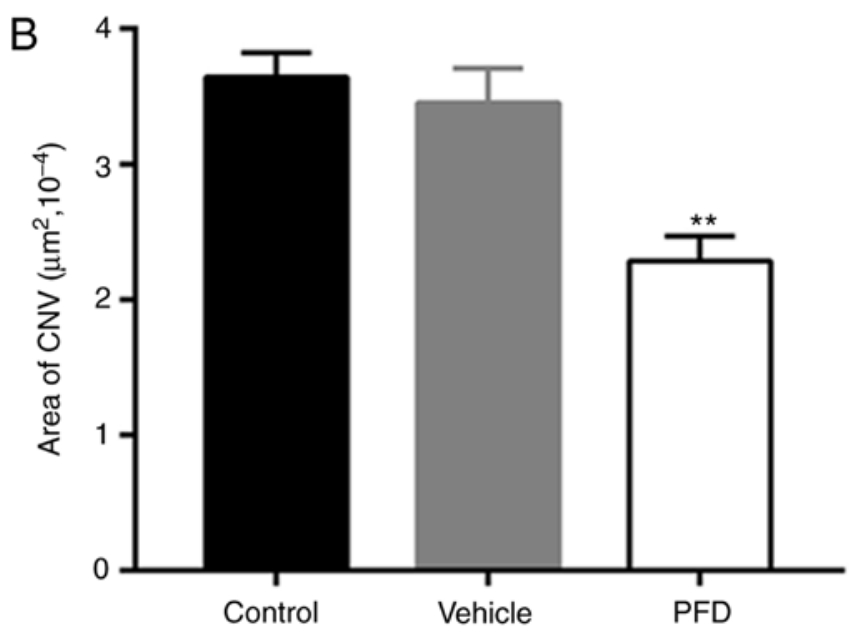

Figure 5. Intravitreal injection of PFD attenuates the formation of CNV. (A) Representative images of isolectin B4 (green) staining of the retinal pigment epithelium-choroid-sclera flat mounts obtained from the control, vehicle and PFD injection groups. Scale bar, $200 \mu \mathrm{m}$. (B) Quantitative measurement of the $\mathrm{CNV}$ area. ${ }^{* *} \mathrm{P}<0.01$ vs. vehicle. $\mathrm{PFD}$, pirfenidone; $\mathrm{CNV}$, choroidal neovascularization.

found to be significantly reduced in the PFD injection group, compared with that measured in the control and vehicle injection groups. This was also observed for VEGF (Fig. 3A and B). This suggested that PFD may inhibit the formation of CNV by downregulating the expression levels of TGF $\beta_{2}$ and VEGF.

PFD alleviates the leakage of CNV. Notably, in the control and vehicle injection groups, the brightness and size of the spot were almost identical (Fig. 4A). Fluorescein angiography showed that the leakage area of $\mathrm{CNV}$ was diminished in the PFD injection 


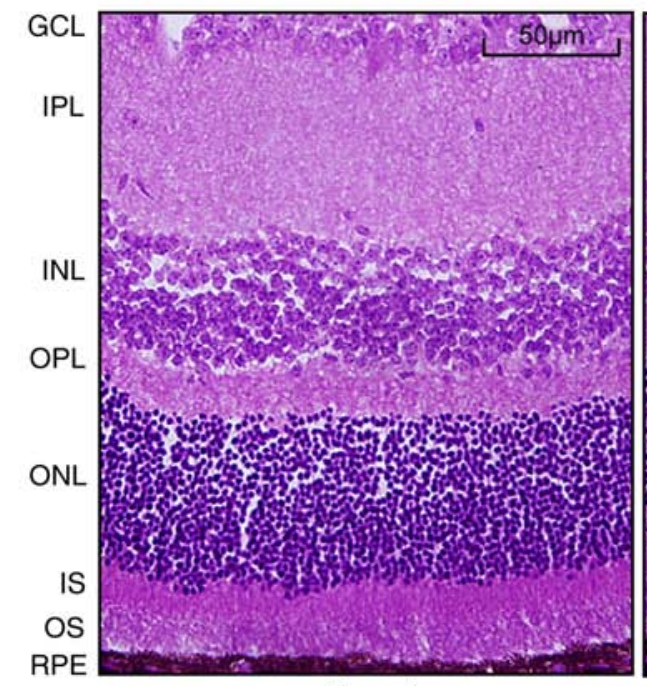

Normal

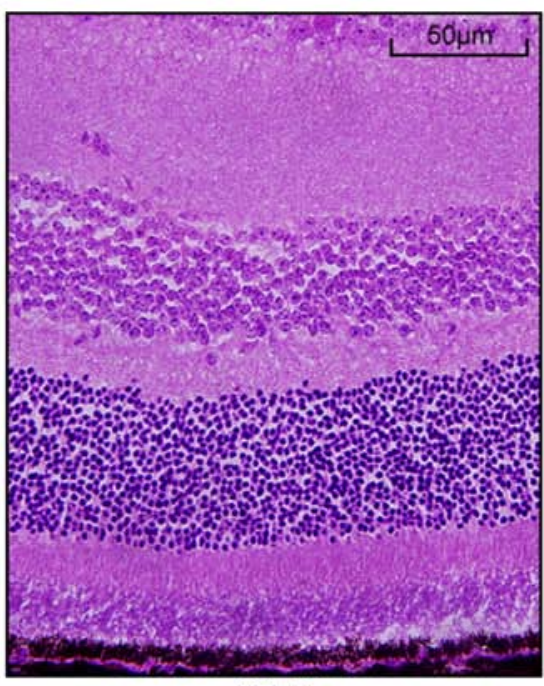

Vehicle

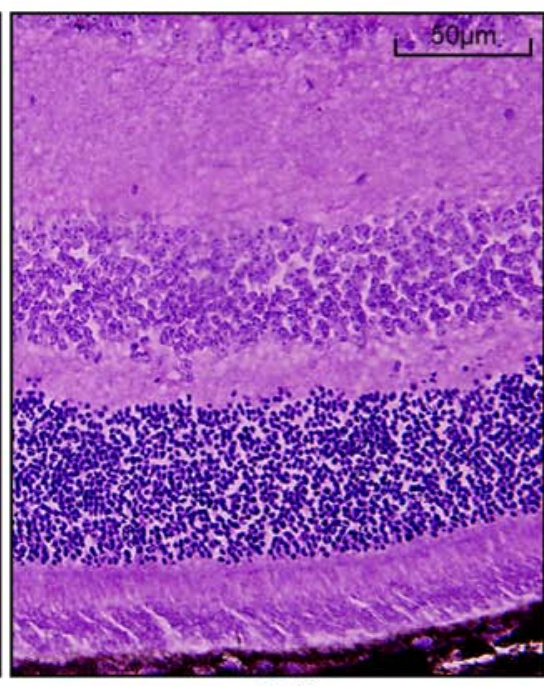

PFD

Figure 6. Intravitreal injection of PFD has no effect on the mouse retina. H\&E staining images of choroidal neovascularization. Retinal and choroidal structure in the normal, vehicle and PFD groups. Scale bar, $50 \mu \mathrm{m}$. RPE, retinal pigment epithelium; OS, outer segment; IS, inner segment; ONL, outer nuclear layer; OPL, outer plexiform layer; INL, inner nuclear layer; IPL, inner plexiform layer; GCL, ganglion cell layer; PFD, pirfenidone.

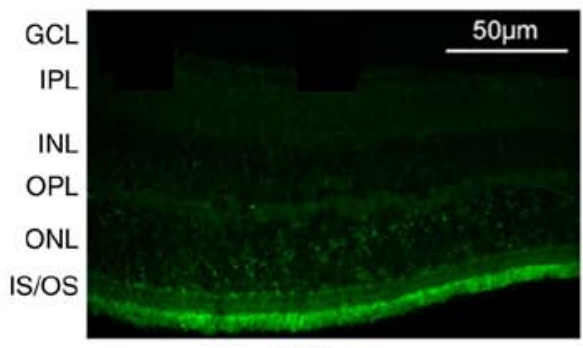

CNV

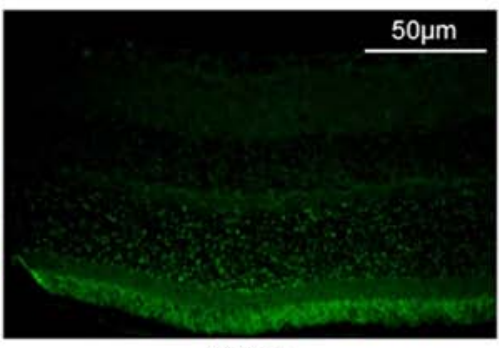

Vehicle

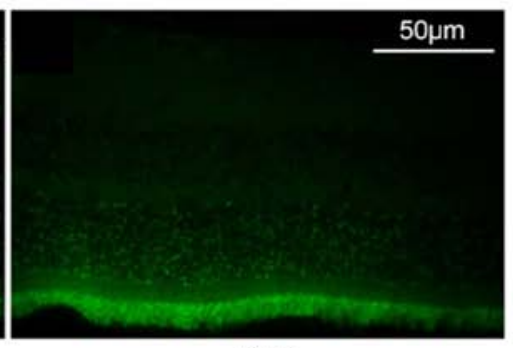

PFD

Figure 7. Intravitreal injection of PFD does not cause apoptosis. TUNEL labeling of the retinas obtained from the control, vehicle and PFD groups. Scale bar, $50 \mu \mathrm{m}$. OS, outer segment; IS, inner segment; ONL, outer nuclear layer; OPL, outer plexiform layer; INL, inner nuclear layer; IPL, inner plexiform layer; GCL, ganglion cell layer; PFD, pirfenidone; $\mathrm{CNV}$, choroidal neovascularization.

group (Fig. 4A). Meanwhile, PFD decreased the number of spots with extensive leakage (score $\geq 2$ ), whereas it increased the number of spots with limited leakage (score 0 or 1; Fig. 4B).

$P F D$ reduces the area of $C N V$. The areas of isolectin $\mathrm{B} 4$ staining, marked with the green fluorescent mass in the image, were notably smaller in the PFD injection group than those observed in the control and vehicle injection groups (Fig. 5A). The difference in the quantitative measurement of the area of CNV formation was also statistically significant (Fig. 5B). Combined with the hypofluorescent leakage of the mouse retina shown on FFA, it was demonstrated that an injection of $0.5 \%$ PFD into the vitreous body of mice after laser photocoagulation could block the formation of $\mathrm{CNV}$ and prevent the development of new blood vessels.

Toxicity of PFD on the mouse retina. To confirm the safety of systemic PFD application, the retina in the region without CNV injury was collected from the PFD group and the histological changes were compared with the tissue from normal animals. Similar to the normal mice, the layers of the neural retina in the region without $\mathrm{CNV}$ in the PFD group were closely organized, well-defined, and the cells were neatly arranged; there were no obvious morphological abnormalities (Fig. 6). Cryosections obtained from the normal, vehicle and PFD groups on day 28 were used for the TUNEL assay to analyze the effect of treatment with PFD on cell death in the mouse retina. There was no obvious difference in the apoptosis rate of the PFD group compared with the vehicle group (Fig. 7). This confirmed that the systemic application of PFD was safe to use on retinal tissue.

\section{Discussion}

In the present study, laser photocoagulation was used to induce a CNV model in male C57BL/6J mice. The immunofluorescent analysis of retinal cryosections showed that the localization of TGF $\beta_{2}$ in the mouse retina changes from the GCL and OPL in the normal retina to the GCL, OPL and IPL in the injured retina. Regarding the effect of PFD on CNV, three dimensions were analyzed to demonstrate that PFD could inhibit the formation of CNV by downregulating the expression of VEGF. Finally, H\&E staining and a TUNEL assay showed that the administration of PFD did not cause damage to the mouse retina.

It is well established that numerous cytokines, such as VEGF, matrix metalloproteinases (MMPs), tissue inhibitor for MMP3 (TIMP3) and TGF 3 , are involved in different stages of 
CNV (32). Specifically, VEGF plays a key role as an inciting stimulus involved in the development of CNV; it triggers the growth of vascular endothelial cells, enhances microvascular permeability and promotes monocyte chemotaxis $(3,13)$. In elderly individuals, the expression levels of MMPs are increased, and responsible for the degradation of the extracellular matrix (ECM) (33). Meanwhile, TIMP3 can inhibit the expression of these MMPs in order to remodel the ECM. As a result, the ratio of MMPs to TIMP3 is essential for ECM turnover, and controls pathology in the Bruch's membrane $(34,35)$. TGF $\beta$ is upregulated during the development of CNV, stimulates the secretion of VEGF, and exerts a strong effect on the process of collagen remodeling and scar contraction (36). In addition, TGF blockages could inhibit angiogenesis and the formation of tissue fibrosis $(7,29)$. Based on the present results, treatment with the TGF $\beta_{2}$ inhibitor PFD hindered the formation and development of $\mathrm{CNV}$.

In the posterior segment of the human eye, TGF $\beta$ isoforms are distributed heterogeneously. TGF $\beta_{2}$ is localized in the connective tissue of large choroidal vessels, outer segment of photoreceptors, microglia, smooth muscle cells and pericytes of superficial retinal blood vessels (37). Ogata et al (38) and Yamamoto et al (39) reported that TGF $\beta_{2}$ at both mRNA and protein levels were detected in the GCL of normal and photocoagulated retinas of rats. In the present study, it was observed that TGF $\beta_{2}$ was mainly expressed in the GCL prior to laser injury, while limited expression was detected in the OPL. During the formation and development of CNV, the total expression of TGF $\beta_{2}$ increased. Interestingly, on days 7 and 28 , the immunolocalization of TGF $\beta_{2}$ was noted in the GCL, OPL and IPL. Moreover, the immunoreaction was stronger in the mouse retina on day 28 compared with day 7 . This finding suggested that $\mathrm{TGF} \beta_{2}$ could affect CNV from the early to late stages.

As mentioned in the introduction, PFD exerts its pharmacokinetic effect by modulating the TNF $\alpha$ and TGF $\beta$ pathways, and inhibiting the differentiation of fibroblasts into myofibroblasts (15). There is a body of evidence related to the anti-fibrotic effects of PFD in vitro and in vivo. Kim et al (40) found that non-toxic concentrations of PFD exert significant anti-fibrotic effects on orbital fibroblasts from patients with thyroid-associated ophthalmopathy. Chowdhury et al (41) indicated that PFD decreased collagen synthesis, prevented myofibroblast formation and improved corneal wound healing. After trabeculectomy surgery, Zhong et al (42) used 0.5\% PFD eye drops to improve postoperative bleb survival. Previously, investigations on human Tenon's fibroblasts demonstrated that PFD could inhibit cell proliferation and migration $(43,44)$. Yang et al (45) revealed that PFD inhibited the TGF $\beta_{2}$-induced proliferation, migration and epithelial-mesenchymal transition of human lens epithelial cells by downregulating the TGF $\beta /$ SMAD signaling pathway. Using a proliferative vitreoretinopathy model, Khanum et al (25) confirmed that PFD prevented fibrotic changes involved in proliferative vitreoretinopathy.

Collectively, the present and previous results indicate that PFD could be a potential treatment of wet AMD, providing an alternative to the current methods used in the clinical setting. Both the present study and a previous study (46) have demonstrated that the local application of PFD can offer protection to the eye. It is proposed that intravenous injection should be carried out to assess the clinical application of the drug. This study used the laser-induced CNV model, which has been shown to be repeatable and stable (26). However, there are a number of limitations of the present study. Firstly, the timeline of CNV inhibition, as well as the effect of PFD when new blood vessels are not formed or after the formation of CNV remains uncertain. Secondly, only the anti-neovascular function of PFD was examined (assays were only performed on day 7 post-induction). In future investigations, its anti-fibrotic functions and effects on the relationship between $\mathrm{TGF}_{2}$ and VEGF will be observed. In addition, this study mainly focused on the mechanisms involved in the protective effects of PFD, which were observed after the induction of CNV.

To conclude, the local administration of PFD reduced the formation of CNV by downregulating the expression of VEGF. This indicated that the use of a TGF $\beta$ inhibitor may be a promising therapy for wet AMD.

\section{Acknowledgements}

Not applicable.

\section{Funding}

This study was supported by Nantong Science and Technology Project (grant no. MS22015085).

\section{Availability of data and materials}

The datasets used and/or analyzed during the current study are available from the corresponding author on reasonable request.

\section{Authors' contributions}

YB, LH, XH, CG, YC, LW and SZ performed the experiments and analyzed the data; YB and YS designed the study and wrote the manuscript. All authors read and approved the manuscript and agree to be accountable for all aspects of the research in ensuring that the accuracy or integrity of any part of the work are appropriately investigated and resolved.

\section{Ethics approval and consent to participate}

All experimental procedures were performed in accordance with the requirements of the Animal Welfare Committee of Nantong University [permit nos. SCXK(Su) 2014-0001 and SYXK(Su)2017-0046]. This study adhered to the Association for Research in Vision and Ophthalmology Statement for the Use of Animals in Ophthalmic and Vision Research. The research protocol for the use of animals was approved by the Center for Laboratory Animals of Nantong University.

\section{Patient consent for publication}

Not applicable.

\section{Competing interests}

The authors declare that they have no competing interests. 


\section{References}

1. Gehrs KM, Anderson DH, Johnson LV and Hageman GS: Age-related macular degeneration-emerging pathogenetic and therapeutic concepts. Ann Med 38: 450-471, 2006.

2. Ambati J and Fowler BJ: Mechanisms of age-related macular degeneration. Neuron 75: 26-39, 2012

3. Campa C, Costagliola C, Incorvaia C, Sheridan C, Semeraro F, De Nadai K, Sebastiani A and Parmeggiani F: Inflammatory mediators and angiogenic factors in choroidal neovascularization: Pathogenetic interactions and therapeutic implications Mediators Inflamm 2010, 2010.

4. Ambati J, Atkinson JP and Gelfand BD: Immunology of age-related macular degeneration. Nat Rev Immunol 13: 438-451, 2013.

5. van Lookeren Campagne M, LeCouter J, Yaspan BL and Ye W: Mechanisms of age-related macular degeneration and therapeutic opportunities. J Pathol 232: 151-164, 2014.

6. Recalde S, Zarranz-Ventura J, Fernández-Robredo $P$ García-Gómez PJ, Salinas-Alamán A, Borrás-Cuesta F, Dotor J and García-Layana A: Transforming growth factor- $\beta$ inhibition decreases diode laser-induced choroidal neovascularization development in rats: $\mathrm{P} 17$ and $\mathrm{P} 144$ peptides. Invest Ophthalmol Vis Sci 52: 7090-7097, 2011.

7. Zarranz-Ventura J, Fernández-Robredo $P$, Recalde S, Salinas-Alamán A, Borrás-Cuesta F, Dotor J and García-Layana A Transforming growth factor-beta inhibition reduces progression of early choroidal neovascularization lesions in rats: P17 and P144 peptides. PLoS One 8: e65434, 2013.

8. Ding D, Li C, Zhao T, Li D, Yang L and Zhang B: LncRNA H19/miR-29b-3p/PGRN axis promoted epithelial-mesenchymal transition of colorectal cancer cells by acting on Wnt signaling. Mol Cells 41: 423-435, 2018.

9. Verrecchia $F$ and Rédini F: Transforming growth factor- $\beta$ signaling plays a pivotal role in the interplay between osteosarcoma cells and their microenvironment. Front Oncol 8: 133, 2018

10. Stewart AG, Thomas B and Koff J: TGF- $\beta$ : Master regulator of inflammation and fibrosis. Respirology 23: 1096-1097, 2018.

11. Kita T, Hata Y, Arita R, Kawahara S, Miura M, Nakao S, Mochizuki Y, Enaida H, Goto Y, Shimokawa H, et al: Role of TGF-beta in proliferative vitreoretinal diseases and ROCK as a therapeutic target. Proc Natl Acad Sci USA 105: 17504-17509, 2008

12. Saika S: TGFbeta pathobiology in the eye. Lab Invest 86: 106-115, 2006.

13. Bressler SB: Introduction: Understanding the role of angiogenesis and antiangiogenic agents in age-related macular degeneration. Ophthalmology 116: S1-S7, 2009.

14. Nagineni CN, Samuel W, Nagineni S, Pardhasaradhi K, Wiggert B, Detrick B and Hooks JJ: Transforming growth factor-beta induces expression of vascular endothelial growth factor in human retinal pigment epithelial cells: Involvement of mitogen-activated protein kinases. J Cell Physiol 197: 453-462, 2003.

15. Yamagami K, Oka T, Wang Q, Ishizu T, Lee JK, Miwa K, Akazawa H, Naito AT, Sakata Y and Komuro I: Pirfenidone exhibits cardioprotective effects by regulating myocardial fibrosis and vascular permeability in pressure-overloaded hearts. Am J Physiol Heart Circ Physiol 309: H512-H522, 2015.

16. Iyer SN, Hyde DM and Giri SN: Anti-inflammatory effect of pirfenidone in the bleomycin-hamster model of lung inflammation. Inflammation 24: 477-491, 2000.

17. Selvaggio AS and Noble PW: Pirfenidone initiates a new era in the treatment of idiopathic pulmonary fibrosis. Annu Rev Med 67: 487-495, 2016.

18. King TE Jr, Bradford WZ, Castro-Bernardini S, Fagan EA, Glaspole I, Glassberg MK, Gorina E, Hopkins PM, Kardatzke D, Lancaster L, et al: A phase 3 trial of pirfenidone in patients with idiopathic pulmonary fibrosis. N Engl J Med 370: 2083-2092, 2014.

19. Noble PW, Albera C, Bradford WZ, Costabel U, du Bois RM Fagan EA, Fishman RS, Glaspole I, Glassberg MK, Lancaster L, et al: Pirfenidone for idiopathic pulmonary fibrosis: Analysis of pooled data from three multinational phase 3 trials. Eur Respir J 47: 243-253, 2016.

20. Sharma K, Ix JH, Mathew AV, Cho M, Pflueger A, Dunn SR, Francos B, Sharma S, Falkner B, McGowan TA, et al: Pirfenidone for diabetic nephropathy. J Am Soc Nephrol 22: 1144-1151, 2011.

21. Lopez-de la Mora DA, Sanchez-Roque C, Montoya-Buelna M, Sanchez-Enriquez S, Lucano-Landeros S, Macias-Barragan J and Armendariz-Borunda J: Role and new insights of pirfenidone in fibrotic diseases. Int J Med Sci 12: 840-847, 2015.
22. Ishikawa K, Kannan R and Hinton DR: Molecular mechanisms of subretinal fibrosis in age-related macular degeneration. Exp Eye Res 142: 19-25, 2016.

23. Liu X, Zhu M, Yang X, Wang Y, Qin B, Cui C, Chen H and Sang A: Inhibition of RACK1 ameliorates choroidal neovascularization formation in vitro and in vivo. Exp Mol Pathol 100: 451-459, 2016.

24. Samuels BC, Siegwart JT, Zhan W, Hethcox L, Chimento M, Whitley R, Downs JC and Girkin CA: A novel tree shrew (Tupaia belangeri) model of glaucoma. Invest Ophthalmol Vis Sci 59: 3136-3143, 2018.

25. Khanum BNMK, Guha R, Sur VP, Nandi S, Basak SK, Konar A and Hazra S: Pirfenidone inhibits post-traumatic proliferative vitreoretinopathy. Eye (Lond) 31: 1317-1328, 2017.

26. Shah RS, Soetikno BT, Lajko M and Fawzi AA: A mouse model for laser-induced choroidal neovascularization. J Vis Exp: e53502, 2015

27. Liu B, Gao J, Lyu BC, Du SS, Pei C, Zhu ZQ and Ma B: Expressions of TGF- $\beta 2$, bFGF and ICAM- 1 in lens epithelial cells of complicated cataract with silicone oil tamponade. Int J Ophthalmol 10: 1034-1039, 2017.

28. Hulin A, Deroanne CF, Lambert CA, Dumont B, Castronovo V, Defraigne JO, Nusgens BV, Radermecker MA and Colige AC: Metallothionein-dependent up-regulation of TGF- $\beta 2$ participates in the remodelling of the myxomatous mitral valve. Cardiovasc Res 93: 480-489, 2012.

29. Wang X, Ma W, Han S, Meng Z, Zhao L, Yin Y, Wang Y and Li J: TGF- $\beta$ participates choroid neovascularization through Smad2/3-VEGF/TNF- $\alpha$ signaling in mice with Laser-induced wet age-related macular degeneration. Sci Rep 7: 9672, 2017.

30. Cai Y, Li X, Wang YS, Shi YY, Ye Z, Yang GD, Dou GR, Hou HY, Yang N, Cao XR and Lu ZF: Hyperglycemia promotes vasculogenesis in choroidal neovascularization in diabetic mice by stimulating VEGF and SDF-1 expression in retinal pigment epithelial cells. Exp Eye Res 123: 87-96, 2014.

31. Ozone D, Mizutani T, Nozaki M, Ohbayashi M, Hasegawa N, Kato A, Yasukawa T and Ogura Y: Tissue plasminogen activator as an antiangiogenic agent in experimental laser-induced choroidal neovascularization in mice. Invest Ophthalmol Vis Sci 57: 5348-5354, 2016.

32. Page-McCaw A, Ewald AJ and Werb Z: Matrix metalloproteinases and the regulation of tissue remodelling. Nat Rev Mol Cell Biol 8: 221-233, 2007.

33. Pittayapruek P, Meephansan J, Prapapan O, Komine M and Ohtsuki M: Role of matrix metalloproteinases in photoaging and photocarcinogenesis. Int J Mol Sci 17, 2016.

34. Ardeljan D and Chan CC: Aging is not a disease: Distinguishing age-related macular degeneration from aging. Prog Retin Eye Res 37: 68-89, 2013.

35. Wong CW, Yanagi Y, Lee WK, Ogura Y, Yeo I, Wong TY and Cheung CMG: Age-related macular Degeneration and polypoidal choroidal vasculopathy in Asians. Prog Retin Eye Res 53: 107-139, 2016.

36. Bai Y, Liang S, Yu W, Zhao M, Huang L, Zhao M and Li X: Semaphorin 3A blocks the formation of pathologic choroidal neovascularization induced by transforming growth factor beta. Mol Vis 20: 1258-1270, 2014

37. Tosi GM, Orlandini M and Galvagni F: The controversial role of TGF- $\beta$ in neovascular age-related macular degeneration pathogenesis. Int J Mol Sci 19, 2018.

38. Ogata N, Yamamoto C, Miyashiro M, Yamada H, Matsushima M and Uyama M: Expression of transforming growth factor-beta mRNA in experimental choroidal neovascularization. Curr Eye Res 16: 9-18, 1997.

39. Yamamoto C, Ogata N, Yi X, Takahashi K, Miyashiro M, Yamada H, Uyama M and Matsuzaki K: Immunolocalization of transforming growth factor beta during wound repair in rat retina after laser photocoagulation. Graefes Arch Clin Exp Ophthalmol 236: 41-46, 1998.

40. Kim H, Choi YH, Park SJ, Lee SY, Kim SJ, Jou I and Kook KH: Antifibrotic effect of Pirfenidone on orbital fibroblasts of patients with thyroid-associated ophthalmopathy by decreasing TIMP-1 and collagen levels. Invest Ophthalmol Vis Sci 51: 3061-3066, 2010.

41. Chowdhury S, Guha R, Trivedi R, Kompella UB, Konar A and Hazra S: Pirfenidone nanoparticles improve corneal wound healing and prevent scarring following alkali burn. PLoS One 8: e70528, 2013

42. Zhong H, Sun G, Lin X, Wu K and Yu M: Evaluation of pirfenidone as a new postoperative antiscarring agent in experimental glaucoma surgery. Invest Ophthalmol Vis Sci 52: 3136-3142, 2011. 
43. Guo X, Yang Y, Liu L, Liu X, Xu J, Wu K and Yu M: Pirfenidone induces G1 arrest in human Tenon's fibroblasts in vitro involving AKT and MAPK signaling pathways. J Ocul Pharmacol Ther 33: 366-374, 2017

44. Na JH, Sung KR, Shin JA and Moon JI: Antifibrotic effects of pirfenidone on Tenon's fibroblasts in glaucomatous eyes: Comparison with mitomycin C and 5-fluorouracil. Graefes Arch Clin Exp Ophthalmol 253: 1537-1545, 2015.

45. Yang Y, Ye Y, Lin X, Wu K and Yu M: Inhibition of pirfenidone on TGF-beta2 induced proliferation, migration and epithlial-mesenchymal transition of human lens epithelial cells line SRA01/04. PLoS One 8: e56837, 2013.
46. Jiang N, Ma M, Li Y, Su T, Zhou XZ, Ye L, Yuan Q, Zhu P, Min Y, Shi W, et al: The role of pirfenidone in alkali burn rat cornea. Int Immunopharmacol 64: 78-85, 2018.

(c) (i) (9) This work is licensed under a Creative Commons cc) ${ }_{\mathrm{EY}}$ NO NO Attribution-NonCommercial-NoDerivatives 4.0 International (CC BY-NC-ND 4.0) License. 